\title{
КОНЦЕПТУАЛЬНАЯ МОДЕЛЬ УПРАВЛЕНИЯ РАЗВИТИЕМ РЕГИОНАЛЬНЫХ СОЦИАЛЬНО-ЭКОНОМИЧЕСКИХ СИСТЕМ
}

\author{
(c) 2021 Родионов Дмитрий Григорьевич \\ доктор экономических наук, профессор, Высшая инженерно-экономическая школа \\ Санкт-Петербургский политехнический университет Петра Великого, Россия, Санкт-Петербург \\ E-mail: dmitry.rodionov@spbstu.ru \\ (C) 2021 Карпенко Павел Алексеевич \\ Высшая инженерно-экономическая школа \\ Санкт-Петербургский политехнический университет Петра Великого, Россия, Санкт-Петербург
}

(C) 2021 Конников Евгений Александрович

кандидат экономических наук, доцент, Высшая инженерно-экономическая школа

Санкт-Петербургский политехнический университет Петра Великого, Россия, Санкт-Петербург

E-mail: konnikov.evgeniy@gmail.com

Состояние региональной социально-экономической системы может быть многомерно представлено вариативной совокупностью показателей, стохастически связанных друг с другом. Система связей индикативных показателей носит крайне комплексный характер, что определяет региональную социально-экономическую систему как крайне комплексный объект управления. Для целей формирования направленного вектора развития региональной социально-экономической системы в рамках данной статьи предлагается концептуальная модель, обладающая центроидом. В качестве центроида концептуальной модели управления развитием региональных социальноэкономических систем выступает совокупность квантификаторов состояния трудовых ресурсов.

Ключевые слова: региональная социально-экономическая система, трудовые ресурсы, квантификация состояния трудовых ресурсов.

Факторная специфика развития региональных социально-экономических систем крайне дифференцированная. Принцип системности восприятия процесса взаимодействия квантификационных компонент региональных социально-экономических систем наиболее наглядно представлен в работе [1]. Предложенная авторами концептуальная модель взаимовлияния показателей социального потенциала региона сущностно охватывает и систематизирует процесс развития региональной социальноэкономической системы, распространяясь за пределы исключительно социального потенциала. Подразделение региональной социальноэкономической системы на субсистемы, позволило выделить пять уникальных, системно связанных макрокомпонент, уровень развития которых проявляется в совокупности количественных показателей. При этом, многоуровневое взаимовлияние, описанное системой регрессионных уравнений позволило сформировать инструментарий прогнозирования условного импульса трансформации всей региональной социально-экономической системы, вследствие целевого направленного воздействия на один или несколько описанных показателей. Однако, именно доказанная авторами исключительная комплексность и комбинационность взаимовлияния рассмотренных показателей определяет вариативность и ограниченность процесса управление развитием. Данная специфика обусловлена следующими тезисами:

1. Структура модели взаимовлияния показателей, отражающих социальный потенциал региональной социально-экономической системы, содержит значимое количество реверсивных связей, что в конечном итоге определяет часть показателей состоянием условной суперпозиции, в рамках которой однонаправленное воздействие на первичный показатель может спровоцировать разнонаправленное самомедиирование данной части показателей. Данное свойство является условным, и в рамках объективного процесса управления развитием региональной социально-экономической системы в первую очередь проявляется в значительном по- 
вышении вариативности конечного результата. Следовательно, представленная модель в большей степени является теоретической и требует упрочнения для целей применения на практике. Однако, уточнение данной модели предполагает отказ от комплексности, что в свою очередь фактически требует исключительно полного переформирования всей разработанной авторами системы регрессионных уравнений.

2. Сформированная модель дифференцирует рассматриваемые показатели исключительно в рамках выделенных авторами региональный социально-экономических субсистем, в то время как данный показатели также могут быть разделены в соответствии с принципом управляемости, на управляемый, частично управляемые и неуправляемые, а также в соответствии с прицепом результативности, на факторные и результирующие. Данное разделение позволяет ограничить процесс управления исключительно практико-ориентированными решениями.

Представленные тезисы определяют вектор развития представленного авторами подхода. Следовательно, наиболее целесообразной концептуальной модификацией описанного подхода к формированию систем управления развитием региональных социально-экономических систем является первичная дифференциация управляемых параметров. Одним из ключевых признаков дифференциации может выступать принцип классификации экономических ресурсов. Наиболее полная классификация экономических ресурсов, как факторов влияния на развитие региональных социально-экономических систем, представлена в рассмотренной ранее работе [2]. В частности автором выделяются пять основных групп - природные факторы, трудовые факторы, финансовые факторы, предпринимательские факторы и факторы знаний. Каждый и представленных групп факторов может выступать в качестве центроида модели управления развитием региональной социальноэкономической системы. Рассмотрим каждый из факторов более детально, применительно к региональным социально-экономическим системам.

Под природными факторами развития региональных социально-экономических систем стоит понимать совокупность благ природного происхождения, находящихся в собственности региона, а также совокупность относительных географических и природных преимуществ ре- гиона. В совокупность благ природного происхождения в свою очередь стоит входят ископаемые ресурсы, лесной и биологический фонд, а также почвенные ресурсы. Под совокупностью относительных географических и природных преимуществ региона стоит понимать относительные выгоды от использования логистической, климатической и иной специфики региона, определенной исключительно свойствами локации. Данная совокупность факторов является исключительно базовым свойством региональной социально-экономической системы, что определяет исключительную ограниченность с точки зрения управления. Несмотря на то, что процесс управления природными ресурсами также крайне комплексный и вариативный с точки зрения потенциальной эффективности, первичные количественные свойства совокупности данных ресурсов крайне дифференцированы в региональном разрезе. Таким образом, использование квантификаторов состояния данных факторов в качестве центроида модели управления развитием региональной социально-экономической системы крайне нецелесообразно, так как в данном случае формируемую модель необходимо специфицировать в соответствии с ресурсной базой каждого конкретного региона.

Далее рассмотрим финансовые факторы. Под финансовыми факторами развития региональных социально-экономических систем стоит понимать совокупность материальных благ, находящихся в собственности региона, регионального бизнеса и региональных домохозяйств. Данную совокупность можно дифференцировать в соответствии с принципом оборачиваемости на основные совокупность основных производственных фондов, совокупность оборотных производственных фондов и совокупность фондов обращения. Данный факторы являются в значительной степени более управляемыми, в сравнении с природными факторами, так как их совокупность формируется исключительно как следствие управленческого воздействия. С концептуальной точки зрения, именно данная ресурсная совокупность определяет объем и качество генерируемого конечного блага, так как сущность именно данные ресурсы трансформируются в объекты конечного блага, а также формируют производственно-технологический базис региона. Однако, необходимо учитывать значимую дифференциацию базисного уровня количественного и качественного состояния 
данной совокупности ресурсов в разрезе региональных социально-экономических систем. Данная дифференциация во многом обусловлена длительным процессом дифференциации векторов развития региональных социальноэкономических систем, как в рамках истории Российской Федерации, так и в рамках истории СССР. Формирование региональные промышленных кластеров в эпоху индустриализации страны определило формирование значимой дифференциации региональных социальноэкономических систем с точки зрения данной ресурсной совокупности, что определяет необходимость спецификации формируемой модели в соответствии с ресурсной базой каждого конкретного региона. Данная проблематика идентична проблематике использования природных факторов в качестве в качестве центроида модели управления развитием региональной социально-экономической системы.

Под предпринимательскими факторами стоит понимать совокупность сформированных в рамках региона предпринимательских структур и институтов, характеризующихся в первую очередь многомерностью опыта коммуникации, организации и управления процессом развития сложных систем. Данная совокупность факторов носит наиболее нематериальный характер, и во многом является вторичной по отношению к трудовым факторам и факторам знаний. В качестве базовых медиаторов предпринимательских факторов выступает в первую очередь региональная политика в области поддержки малого и среднего бизнеса, а также наличие материальной и природной базы для развития крупного бизнеса. Таким образом данную совокупность факторов можно назвать вторичной по отношению к ранее описанным совокупностям, однако, именно предпринимательские факторы являются основой эффективного использования ресурсного базиса. Описанное реверсивное свойство взаимодействия представленных факторных совокупностей во многом определяет диалектический характер развития региональных социально-экономических систем. Таким образом, использование предпринимательских факторов в качестве центроида модели управления развитием региональной социальноэкономической системы неэффективно.

Под факторами знаний следует понимать совокупность сформированных в рамках региональной социально-экономической системы институтов генерации и развития объектов интеллектуальной собственности, а также институтов распространения и развития знаний, умений и навыков в разрезе основных направлений. Данная совокупность факторов первую очередь представлена учреждениями высшего образования, научно-исследовательскими институтами и отдельными научными школами. Именно данная факторная совокупность определяет процесс формирования научно-исследовательского базиса, являющегося основой для формирования, интеграции, оптимизации и развития решений прикладного характера. Также, необходимо отметить, что данная факторная совокупность, несмотря на доступность инструментов внутренней миграции, во многом определяет развитие трудовых ресурсов. Однако, данная процесс медиирования факторов знаний носит предиктивный характер исключительно в долгосрочной перспективе, так как процесс формирования научной школы, последующее достижение значимых исследовательских результатов и формирование на их основе прикладных решений крайне дифференцировано во времени. При этом, необходимо учитывать неизменность высоко-конкурентной среды в рамках данной факторной совокупности, что в совокупности с долгосрочным и волотильным характером результатов определяет сравнительно низкий уровень целесообразности использования факторов знаний в качестве центроида модели управления развитием региональной социальноэкономической системы неэффективно.

В завершении необходимо рассмотреть трудовые факторы. Под трудовыми факторами стоит понимать совокупность зарегистрированного в рамках региональной социальноэкономической системы трудоспособного населения. В первую очередь необходимо отметить, что данный ресурс является крайне динамически трансформируемым и во много определяющим системное взаимодействие описанных ранее ресурсных совокупностей. Данная специфика определяет возможность индексации последствий процесса управления развитием региональной социально-экономической системы посредствам квантификации сравнительной трансформации именно трудовых факторов. При этом сущностно являясь основой формирования социальной среды, именно конверсия последствий процесса управления развитием региональной социально-экономической системы 
в трансформацию трудовых факторов определяет текущее состояние социальной среды, которая в свою очередь является базисом экономического развития в долгосрочной перспективе. Таким образом, именно состояние трудовых ресурсов региона, выраженное через совокупность трудовых факторов, определяет конверсию последствий процесса управления развитием региональной социально-экономической системы в ее результаты. Следовательно, совокупность трудовых факторов является наиболее эффективным центроидом модели управления развитием региональной социально-экономической системы.

Использование трудовых факторов как центроида формируемой модели предполагает формирование системы факторов входного воздействия и системы результатов конверсии входного воздействия. Под системой факторов входного воздействия следует понимать совокупность квантифицируемых переменных, являющихся экзогенными по отношению к трудовым факторам. При этом необходимо отметить, что неизменно обязательным свойством данных факторов должен быть полностью или частично управляемый характер. Дифференцировать данные факторы целесообразно в соответствии с описанной ранее спецификой дифференциации экономических ресурсов. Таким образом, в первую очередь могут быть выделены природные факторы. В качестве управляемого показателя, характеризующего воздействие субъектов управления на развитие совокупности природных факторов региональной социальноэкономической системы можно выделить инвестиции в основной капитал, направленные на охрану окружающей среды и рациональное использование природных ресурсов. Данный показатель дифференцируется в соответствии с направлениями инвестирования, а именно инвестиции в охрану атмосферного воздуха, инвестиции в охрану и рациональное использование водных ресурсов, а также инвестиции в строительство и обслуживание станций для очистки сточных вод. Выделенная совокупность направлений инвестирования не является исчерпывающей, однако они составляют большую часть от всей совокупности. Приращение данных параметров в среднесрочной и долгосрочной перспективе оказывает воздействие на повышение уровня экологической безопасности региона, что в свою очередь неизменно положительно сказывается на общем усредненном состоянии физического и морального здоровья населения. Представленная формально-логическая связь определяет конверсию процесса приращения инвестиций в основной капитал, направленных на охрану окружающей среды и рациональное использование природных ресурсов, в изменение состояния трудовых ресурсов, что в свою очередь потенциально может оказывать влияние на общее развитие региональной социальноэкономической системы. Помимо выделенного показателя инвестиций, неизменно оказывающего положительное воздействие на состояние трудовых ресурсов, необходимо выделить показали, отражающие негативное воздействие на природные ресурсы региона. Условие необходимой полной или частичной управляемости определяет техногенный характер данных показателей. Таким образом, наиболее целесообразным к использованию в данном случае выступает объем выбросов вредных (загрязняющих) веществ. Данный показатель является многомерно управляемым, что определяется как наличием возможности снижения за счет сокращения объёмов производства продукции, технологический процесс производства которой сопровождается значимыми выбросами загрязняющих веществ, так и возможностью компенсации данного воздействия за счет совершенствования систем отчистки и компенсации выбрасываемых загрязняющих веществ. Воздействие изменения данного показателя на состояние трудовых ресурсов носит реверсивный характер, что определяет необходимость его снижения. Совокупность приведенных показателей является необходимой и достаточной для целей описания влияния природных факторов на трудовые в рамках развития региональной социально-экономической системы, так как иные природные факторы не являются полностью или частично управляемыми, что позволит сформировать на их основе исключительно оценочную модель, непригодную для целей управления развитием региональных социально-экономических систем.

Далее необходимо рассмотреть совокупность факторов, многомерно описывающих состояние материальных ресурсов, формирующих внутрипроизводственную и внешнюю среду. Данная совокупность может быть условно разделена на производственные факторы и инфраструктурные факторы. Сущность производственных факторов определяется состоянием 
материальных ресурсов, используемых в рамках процесса генерации блага. Данная совокупность ресурсов может быть разделена в соответствии с признаком оборачиваемости, а также в соответствии с характером участия в производственном процессе. Общее сравнительное состояние основных производственных фондов может быть описано показателем использования производственных мощностей. Также, условно целесообразным может являться использования в качестве анализируемого показателя входного влияния величины накопленной амортизации, однако, данный показатель обладает значимой отраслевой спецификой, что определяет необходимость отраслевой спецификации модели, что в свою очередь противоречит целеполаганию данного исследования. Показатель использования производственных мощностей измеряется в процентах и сущностно отражает долю активно задействованного производственного потенциала региона. Данный показатель является исключительно управляемым и во многом может медиироваться субъектами управления региональной социально-экономической системы как напрямую, посредствам размещение государственного заказа, так и косвенно, посредствам формирования особых условий функционирования региональных предприятий. Приращение данного показателя оказывает прямое воздействие на состояние трудовых факторов, в первую очередь за счет насыщения регионального рынка труда предложением, а также формирования активной экономической среды. Также в качестве комплексного фактора производственного характера стоит рассматривать ВРП на душу населения. Данный показатель носит дуалистичный характер, и во много является результирующим. Однако, в современных условиях развития региональных социальноэкономических систем комплексный результат, которым непосредственно является ВРП на душу населения, определяется не столько условной эффективностью трудовых ресурсов, сколько эффективностью, адаптивностью и предиктивностью процесса его формирования, как на технологическом, так и на административноуправленческом уровнях. При этом именно ВРП на душу населения сущностно отражает уровень благосостояния населения, что в свою очередь напрямую влияет на состояние трудовых факторов. Таким образом, ВРП на душу населения наиболее целесообразно использовать именно в качестве показателя входного влияния.

Параллельно развитию производственной среды, комплексное развитие региональных социально-экономических систем неизменно предполагает развитие инфраструктуры, как связующего межпроизводственного ресурса. Деградация и неудовлетворительное состояние региональной инфраструктуры определяет повышение логистических издержек, что в свою очередь приводит к повышению трудоемкости взаимодействия населения и бизнеса как в рамках процесса производства, так и в рамках процесса потребления. В качестве показателей, отражающих состояние инфраструктурных факторов, наиболее целесообразно выделить показатель наличия автомобильного транспорта, дифференцированного на общественный (в частности автобусы, как наиболее универсальный вид общественного транспорта) и частный (в частности легковые автомобили). Также, данный показатель может быть дополнен показателем протяжённости автомобильных дорого общественного пользования.

Представленная совокупность факторов входного влияния потенциально может быть дополнена совокупностью показателей, отражающих состояние системы здравоохранения, образования и иными показателями социального характера. В рамках данного исследования в качестве показателей, отрезающих состояние социальной среды было решено использовать число учащихся в общеобразовательных учреждениях, охваченных питанием, долю учреждений здравоохранения, использующих сеть интернет, в общем числе учреждений здравоохранения, а также реальную начисленную заработную плату в процентах к соответствующему периоду предыдущего года. Приведенная совокупность показателей входного влияния представлена в таблице 1.

Представленная совокупность показателей в достаточной мере описывает входное влияние региональной социально-экономической системы на состояние трудовых ресурсов. Далее необходимо рассмотреть совокупность результирующих по отношению к трудовому ресурсу показателей. Данная совокупность в первую очередь определяется параметрами, характеризующими среду социального взаимодействия. Данная среда в первую очередь проявляется в совокупности показателей, отражающих уровень социальной безопасности, такими как ко- 
Таблица 1. Совокупность показателей входного влияния

\begin{tabular}{|c|c|c|c|}
\hline № & Наименование показателя & Условное обозначение & Единицы измерения \\
\hline \multicolumn{4}{|c|}{ Природные факторы } \\
\hline 1. & $\begin{array}{l}\text { Объем выбросов вредных (загрязняющих) ве- } \\
\text { ществ в атмосферный воздух от автомобильного } \\
\text { транспорта }\end{array}$ & $N_{1}$ & тонн \\
\hline 2. & $\begin{array}{l}\text { Инвестиции в основной капитал, направленные } \\
\text { на охрану окружающей среды и рациональное } \\
\text { использование природных ресурсов (охрана ат- } \\
\text { мосферного воздуха) }\end{array}$ & $N_{2}$ & тыс. руб. \\
\hline 3. & $\begin{array}{l}\text { Инвестиции в основной капитал, направленные } \\
\text { на охрану окружающей среды и рациональное } \\
\text { использование природных ресурсов (охрана и } \\
\text { рациональное использование водных ресурсов) }\end{array}$ & $N_{3}$ & тыс. руб. \\
\hline \multicolumn{4}{|c|}{ Производственные факторы } \\
\hline 1. & $\begin{array}{l}\text { Уровень использования производственных мощ- } \\
\text { ностей }\end{array}$ & $P_{1}$ & $\%$ \\
\hline 2. & ВПР на душу населения & $P_{2}$ & тыс. руб. \\
\hline \multicolumn{4}{|c|}{ Инфраструктурные факторы } \\
\hline 1. & Наличие автомобильного транспорта (автобусов) & $I_{1}$ & единиц \\
\hline 2. & $\begin{array}{l}\text { Наличие автомобильного транспорта (легкового } \\
\text { автотранспорта) }\end{array}$ & $I_{2}$ & единиц \\
\hline 3. & $\begin{array}{l}\text { Протяженность автомобильных дорог общего } \\
\text { пользования }\end{array}$ & $I_{3}$ & KM. \\
\hline \multicolumn{4}{|c|}{ Социальные факторы } \\
\hline 1. & $\begin{array}{l}\text { Число учащихся в общеобразовательных учрежде- } \\
\text { ниях, охваченных питанием }\end{array}$ & $S_{1}$ & человек \\
\hline 2 . & $\begin{array}{l}\text { Доля учреждений здравоохранения, использую- } \\
\text { щих сеть Интернет, в общем числе учреждений } \\
\text { здравоохранения }\end{array}$ & $S_{2}$ & $\%$ \\
\hline 3. & $\begin{array}{l}\text { Реальная начисленная заработная плата в про- } \\
\text { центах к соответствующему периоду предыдуще- } \\
\text { го года }\end{array}$ & $S_{3}$ & $\%$ \\
\hline
\end{tabular}

личество правонарушений в разрезе основных статей уголовного кодекса, а также в совокупности показателей, отражающих условное «оздоровление» социума, такими как объем потребляемых алкогольных напитков и наркотических веществ. Также, необходимо отдельно отметить увеличение уровня безработицы, как следствие реверсивной конверсии приращения основных показателей входного влияния. Совокупность результирующих показателей представлена в таблице 2.

Приведенная совокупность показателей может быть агрегирована в рамках единой концептуальной модели. Данная модель представлена на рисунке 1.

Как можно видеть, центроидом концепту- альной модели управления развитием региональных социально-экономических систем выступает совокупность квантификаторов состояния трудовых ресурсов. Данные квантификаторы могут быть агрегированы на основе анализа сравнительного состояния коммуникативных проявлений населения региона. Данный тезис основан на предположении о связи психологического состояния представителей социума с результатами их профессиональной деятельности и иными социальными проявлениями. Таким образом, для целей оценки состояния трудовых ресурсов необходимо квантифицировать коммуникативную среду, чему непосредственно посвящен последующий раздел данного исследования. 
Таблица 2. Совокупность результирующих показателей

\begin{tabular}{|c|l|c|c|}
\hline № & \multicolumn{1}{|c|}{ Наименование показателя } & Условное обозначение & Единицы измерения \\
\hline 1. & $\begin{array}{l}\text { Количество преступлений, зарегистриро- } \\
\text { ванных в отчетном периоде по ст.105 УК РФ } \\
\text { (Убийство) }\end{array}$ & $\boldsymbol{R}_{\mathbf{1}}$ & единиц \\
\hline 2. & $\begin{array}{l}\text { Количество преступлений, зарегистриро- } \\
\text { ванных в отчетном периоде по ст.111 УК РФ } \\
\text { (Умышленное причинение вреда здоровью) }\end{array}$ & $\boldsymbol{R}_{\mathbf{2}}$ & единиц \\
\hline 3. & $\begin{array}{l}\text { Количество преступлений, зарегистриро- } \\
\text { ванных в отчетном периоде по ст.131 УК РФ } \\
\text { (Изнасилование) }\end{array}$ & $\boldsymbol{R}_{\mathbf{3}}$ & единиц \\
\hline 5. & $\begin{array}{l}\text { Количество преступлений, зарегистриро- } \\
\text { ванных в отчетном периоде по ст.213 УК РФ } \\
\text { Хулиганство) }\end{array}$ & $\boldsymbol{R}_{\mathbf{4}}$ & человек \\
\hline 5. & $\begin{array}{l}\text { Число умерших по основным классам и } \\
\text { отдельным причинам смерти в расчете на } \\
\text { 100000 (Самоубийство) }\end{array}$ & $\boldsymbol{R}_{\mathbf{5}}$ & человек \\
\hline 6. & $\begin{array}{l}\text { Число умерших по основным классам и } \\
\text { отдельным причинам смерти в расчете на } \\
\text { 100000 (Случаи отравления алкоголем) }\end{array}$ & $\boldsymbol{R}_{\mathbf{6}}$ & человек \\
\hline 7. & $\begin{array}{l}\text { Общая численность безработных в соответ- } \\
\text { ствии с методологией МОТ }\end{array}$ & $\boldsymbol{R}_{7}$ & \\
\hline
\end{tabular}

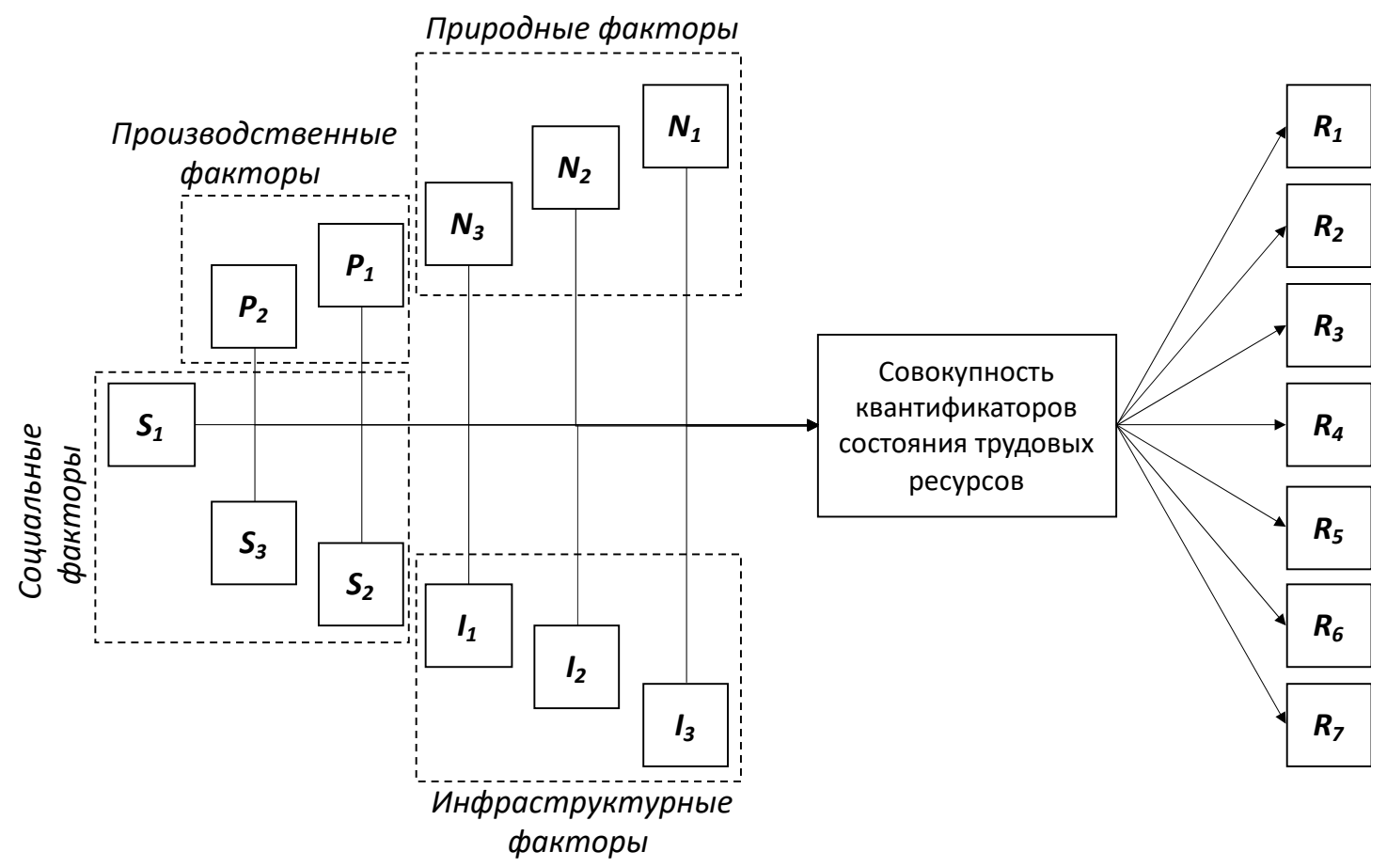

Рисунок 1. Концептуальная модель управления развитием региональных социально-экономических систем 


\section{Библиографический список}

1. Родионов Д.Г., Баранова И. В., Насрутдинов М.Н. Формирование модели интерферирования показателей социального потенциала региона // Российский экономический интернет-журнал. 2019. № 4. С. 119

2. Кисуркин А.А. Факторы, влияющие на инновационное развитие региона и их классификация по уровням управления // Современные проблемы науки и образования. Общество с ограниченной ответственностью» Издательский Дом» Академия, 2012. № 2. С. 294.

3. Белостоцкая А.А., Забелин Б. Ф., Конников Е.А., Мокейчев Е.В. Содержательный аспект тенденции к отражению действительного результата управления // Экономические науки. 2016. № 136. С. 42-46. 